\title{
Relay Scheduling and Interference Cancellation for Quantize-Map-and-Forward Cooperative Relaying
}

\author{
M. Jorgovanovic, M. Weiner, D. Tse and B. Nikolić \\ School of EECS \\ University of California at Berkeley \\ Berkeley, California, USA \\ \{milos, mgweiner, dtse, bora $\} @$ eecs.berkeley.edu
}

\author{
I. -H. Wang \\ School of CCS \\ EPFL \\ Lausanne, Switzerland \\ i-hsiang.wang@epfl.ch
}

\author{
V. Nagpal \\ Nokia Research Center, Berkeley \\ Nokia Corporation \\ Berkeley, California, USA \\ vinayak.nagpal@nokia.com
}

\begin{abstract}
This paper presents system design aspects of a multirelay half-duplex QMF cooperative system. We propose two simple algorithms that address the design of relay scheduling and inter-relay interference schemes. Proposed linear-complexity scheduling algorithm is proven to be optimal for a multi-relay diamond network under specific channel conditions. We demonstrate through simulations that for typical channel conditions, the achievable QMF rate of a five-relay cooperative system is up to 3 times higher compared to a system without cooperation.
\end{abstract}

\section{INTRODUCTION}

Cooperative relaying has been envisioned as a promising technique for improving the spectral efficiency of a wireless transmission. There has been a lot of progress in understanding different aspects of cooperation, and several cooperation schemes have been proposed. We focus on a quantize-mapand-forward (QMF) scheme, which has been shown to perform close to capacity for an arbitrary number of relays [1]. We extend the design aspects of a single-relay QMF system from [2] to a multi-relay QMF system by suggesting simple algorithms that address two fundamental problems for multi-relay cooperation: relay scheduling and inter-relay interference.

We assume an uplink cooperative wireless network with an information source, a destination, and several half-duplex relays close to the source, available for physical-layer cooperation. Relays are assumed to use time-division-duplexing: they listen to the source for some fraction of total time (listening phase), process the received signal, and then forward it to the destination in the remaining fraction (transmit phase). The QMF scheme states that relays process received information by quantizing the received signal at the noise level and then mapping those quantized bits into a random codeword.

For a cooperative network with multiple half-duplex relays, determining the optimal listen/transmit fractions becomes an important factor in achieving the maximum rate of communication. The complexity of this optimization problem grows exponentially with the number of relays $N$. Brahma et al. [3] conjecture that optimal scheduling for a half-duplex diamond network requires at most $N+1$ out of $2^{N}$ network states. They do not, however, provide a general algorithm to choose those states and calculate the schedules. Wang et al. [4] suggest simple heuristics for relay scheduling for decode-and-forward (DF) cooperation scheme that is known not to perform within a constant gap from capacity for a multiple-relay network.

In this paper, we bring insights about relay scheduling for the QMF scheme and a more general cooperative network (Figure 1). Performing scheduling analysis of this network turns out to be difficult. We show that with the suggested system design choices, the general network can be approximated with a diamond network, which further motivates our analysis of relay scheduling for a diamond network. We suggest a local scheduling algorithm, a linear-complexity alternative to an optimal relay scheduling algorithm. We prove that this simple algorithm achieves optimal performance of a diamond network under specific channel conditions.

Another important issue to consider in a multiple-relay cooperative system is interference between relays. Even though signals from other relays can be considered as side information, we constrain the analysis to a single-hop relaying scenario and choose to treat these signals as interference. We use the same system design as described in [2], and propose a simple algorithm for inter-relay interference cancellation. Restricted to linear encoding schemes at the source and the relays [2], we show that the achievable QMF rate of this algorithm applied to the general network from Figure 1 is the same as the achievable QMF rate of the same network without relay-torelay links. We simulate typical channel conditions with these two networks, and demonstrate that the performance of the proposed algorithm is within $5 \%$ of the optimal performance for the general network.

\section{Relay Scheduling for CoOperative Networks}

\section{A. System Description}

The cooperative wireless network that we consider in this paper consists of a single-antenna source $S, N$ half-duplex single-antenna relays $R_{1}, \ldots, R_{N}$, and a multiple-antenna destination $D$ (Figure 1). Let $\mathcal{R}$ denote a set of indices of all relays in the system, i.e. $\mathcal{R}=\{1, \ldots, N\}$. We assume a Gaussian network model, with the channels between the terminals described as scalars $h_{T_{1} T_{2}}$ and vectors $\underline{h}_{T_{1} D}$, for $T_{1}, T_{2} \in\left\{S, R_{1}, \ldots, R_{N}\right\}$.

We assume that communication between source and destination occurs in frames, similar to an actual communication system. Let $f_{i}$ be a fraction of the frame length for which relay 

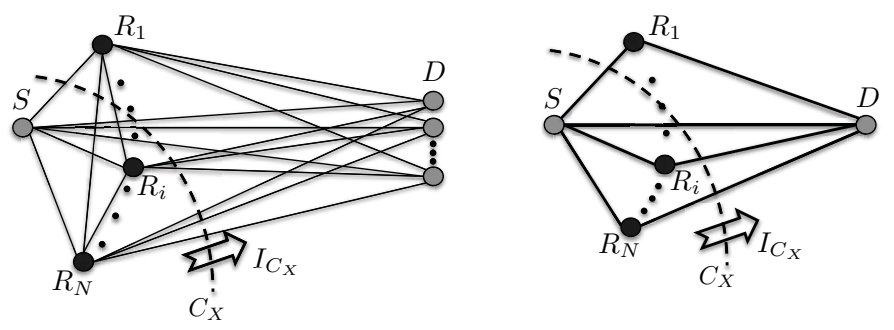

Fig. 1. General network model (left) and diamond network model (right)

$R_{i}$ is in the listening phase. With $N$ relays in the system, we can partition the frame into $2^{N}$ time fractions as:

$$
1=\lambda_{\emptyset}+\sum_{i \in \mathcal{R}} \lambda_{\{i\}}+\sum_{i, j \in \mathcal{R}} \lambda_{\{i, j\}}+\ldots+\lambda_{\mathcal{R}},
$$

where $\lambda_{\mathcal{S}}$ denotes the time fraction that corresponds to network state $\mathcal{S}$, and $\mathcal{S}$ denotes the set of indices of the relays that are in the listening phase. The scheduling problem involves finding the optimal vector $\Lambda$ of these time fractions, $\Lambda=\left[\lambda_{\emptyset}, \lambda_{\{1\}}, \ldots, \lambda_{\{N\}}, \lambda_{\{1,2\}}, \ldots, \lambda_{\{1,2,3\}}, \ldots, \lambda_{\mathcal{R}}\right]$, under condition (1).

\section{B. Relay Scheduling for the General Network}

We calculate the QMF achievable rate of the general cooperative wireless network (Figure 1) in two steps. First, we identify all the cuts, $C_{1}, C_{2}, \ldots, C_{2^{N}}$, in the network. Then we calculate the QMF information flow through each cut, $I_{C_{X}}$, and find the minimum one to be the achievable QMF rate: $R_{Q M F}=\min _{C_{X}}\left\{I_{C_{X}}\right\}$.

Let $\mathcal{R}_{C_{X}}$ and $\mathcal{L}_{C_{X}}$ be the sets of indices of relay nodes on the right and the left side of the cut $C_{X}$, respectively, such that $D \in \mathcal{R}_{C_{X}}$ and $S \in \mathcal{L}_{C_{X}}$. Given the network state $\mathcal{S}$, let $\mathcal{S}_{C_{X}}^{R}$ and $\mathcal{T}_{C_{X}}^{R}$ be the sets of indices of relay nodes on the right side of the cut $C_{X}$ that are in listening and transmitting phase, respectively: $\mathcal{R}_{C_{X}}=\mathcal{S}_{C_{X}}^{R} \cup \mathcal{T}_{C_{X}}^{R}$. Analogously, $\mathcal{L}_{C_{X}}=$ $\mathcal{S}_{C_{X}}^{L} \cup \mathcal{T}_{C_{X}}^{L}$.

It has been shown in [5] that quantization at the noise level at the relays has twofold negative effect on the QMF information flow, $I_{C_{X}}$, compared to the cut-set bound: a) the SNR on $T_{i}-R_{i}$ links, where $T_{i} \in\left\{S, R_{1}, \ldots, R_{N}\right\}$, is reduced by a factor of 2 ; b) for each relay with index $i \in \mathcal{L}_{C_{X}}$, there is an information loss that equals $f_{i}$.

The optimal relay scheduling for the general network model from Figure 1 is found by maximizing the achievable rate:

$$
\Lambda^{*}=\arg \max _{\sum_{\mathcal{S}} \lambda_{\mathcal{S}}=1} \min _{C_{X}}\left\{I_{C_{X}}(\Lambda)\right\}
$$

where $I_{C_{X}}(\Lambda)$ is QMF information flow through the cut $C_{X}$, given a scheduling vector $\Lambda$ that satisfies condition (1). This optimization is done over $2^{N}$ scheduling parameters $\lambda_{\mathcal{S}}$, so its complexity grows exponentially with the number of relays $N$. With a Gaussian network model, this information flow can be expressed as a summation over all possible $\mathcal{S} \subset \mathcal{R}$ :

$$
I_{C_{X}}(\Lambda)=\sum_{\mathcal{S} \subset \mathcal{R}} \lambda_{\mathcal{S}} \log \operatorname{det}\left(I+H_{\mathcal{S}} H_{\mathcal{S}}^{H}\right)-\sum_{i \in \mathcal{L}_{C_{X}}} f_{i} .
$$

$\mathcal{S}$ represents the set of indices of all relays that are listening, i.e. $\mathcal{S}=\mathcal{S}_{C_{X}}^{R} \cup \mathcal{S}_{C_{X}}^{L}$. The logarithm term is the MIMO capacity through the cut $C_{X}$ when the network is in this state. All logarithms are base 2. MIMO capacity is calculated using channel matrix $H_{\mathcal{S}}$ :

$$
H_{\mathcal{S}}=\left[\begin{array}{cc}
\underline{h}_{S D} & H_{\mathcal{T}_{C_{X}}^{L} D} \\
\frac{\underline{h}_{S \mathcal{S}_{C_{X}}^{R}}}{\sqrt{2}} & \frac{H_{\mathcal{T}_{C_{X}}^{L} \mathcal{S}_{C_{X}}^{R}}}{\sqrt{2}}
\end{array}\right],
$$

where $\underline{h}_{S \mathcal{S}_{C_{X}}^{R}}$ is a vector consisting of the channel coefficients between source $S$ and relays with indices in $\mathcal{S}_{C_{X}}^{R}, H_{\mathcal{T}_{C_{X}}^{L} D}$ is a matrix whose columns are channel vectors between the relays with indices in $\mathcal{T}_{C_{X}}^{L}$ and the destination $D$, and $H_{\mathcal{T}_{C_{X}}^{L} \mathcal{S}_{C_{X}}^{R}}$ is a matrix of channel coefficients $h_{R_{i} R_{j}}$ where $i \in \mathcal{T}_{C_{X}}^{L}$, and $j \in \mathcal{S}_{C_{X}}^{R} \cdot \sqrt{2}$ factors are due to the quantization loss at relay $R_{j}$, where $j \in \mathcal{S}_{C_{X}}^{R}$.

\section{Relay Scheduling for the Diamond Network}

For the diamond network in Figure 1, we assume that channels between the terminals are represented through the SNR values $S N R_{S T}$ for $T \in\left\{R_{1}, \ldots, R_{N}, D\right\}$ and $S I N R_{T D}$ for $T \in\left\{R_{1}, \ldots, R_{N}\right\}$.

Let us first assume that the diamond network has only one relay, $R_{1}$. This is a simple scenario when the optimal listening time of the relay is given by (5), which is calculated by balancing the cuts as in [2].

For the diamond network with $N$ relays, the complexity of optimization is exponential with the number of relays. Even though [3] suggests that the number of non-zero scheduling parameters is only $N+1$, there is no systematic way to identify these parameters and calculate the schedules but to perform an optimization over all $2^{N}$ parameters. This is not practical for two reasons: a) the computation may be intense and possibly infeasible in real-time for a large number of relays and fast-fading channels; b) an optimal scheduling may require some relays to switch between $\mathrm{Rx} / \mathrm{Tx}$ mode multiple times per frame. Since each switching instance has some time loss associated with it, it is more practical to make this switch happen only once per frame. Before we propose an actual scheme that satisfies these conditions, we prove the following theorem.

Theorem 1: If listening time of each relay, $f_{i}^{*}$, is chosen according to (5), and the condition:

$$
f_{1}^{*}+f_{2}^{*}+\ldots+f_{N}^{*} \leq 1
$$

holds, then an optimal schedule is given by:

$$
\begin{aligned}
\lambda_{\{i\}}^{*} & =f_{i}^{*}, \forall i \in\{1,2, \ldots, N\} \\
\lambda_{\emptyset}^{*} & =1-\sum_{i=1}^{i=N} f_{i}^{*} \\
\lambda_{\mathcal{S}}^{*} & =0, \forall \mathcal{S},|\mathcal{S}| \geq 2 .
\end{aligned}
$$




$$
f_{i}=\frac{\log \left(1+S I N R_{R_{i} D}\right)}{\log \left(1+S I N R_{R_{i} D}\right)+\left[\log \left(1+S N R_{S D}+\frac{S N R_{S R_{i}}}{2}\right)-\log \left(1+S N R_{S D}\right)+1\right]}
$$

Proof. For the diamond network with $N$ relays, (3) simplifies to (8). It is convenient to rewrite part of the second term in (8) by using $f_{i}$ as:

$$
f_{i}=\lambda_{\{i\}}+\sum_{j \in \mathcal{R}} \lambda_{\{i, j\}}+\sum_{j, k \in \mathcal{R}} \lambda_{\{i, j, k\}}+\ldots+\lambda_{\mathcal{R}}
$$

We can now manipulate (8) into (10), where:

$$
\begin{aligned}
\Delta_{\mathcal{S}_{C_{X}}^{R} \cup \mathcal{S}_{C_{X}}^{L}} & =\sum_{i \in \mathcal{S}_{C_{X}}^{R}} \log \left(1+S N R_{S D}+\frac{S N R_{S R_{i}}}{2}\right) \\
& -\log \left(1+S N R_{S D}+\sum_{i \in \mathcal{S}_{C_{X}}^{R}} \frac{S N R_{S R_{i}}}{2}\right) \\
& -\left(\left|\mathcal{S}_{C_{X}}^{R}\right|-1\right) \log \left(1+S N R_{S D}\right)
\end{aligned}
$$

is strictly non-negative for $\left|\mathcal{S}_{C_{X}}^{R}\right| \geq 2$ and positive values of SNRs because $\prod_{i=1}^{k}\left(x+y_{i}\right)>\left(x+\sum_{i=1}^{k} y_{i}\right) x^{k-1}$. From (10) we conclude that given listening fractions $f_{i}$ for each relay $R_{i}$, overlaps in listening intervals $\left(\lambda_{\{i, j\}}, \lambda_{\{i, j, k\}}\right.$ etc.) always decrease the information flow through an arbitrary cut $C_{X}$. Furthermore, since listening fractions $f_{i}^{*}$ are chosen according to (5), the sum of the first four terms in (10) is the same across all cuts. The fifth term is the penalty term if more than one relay is listening at the same time.

Next we show that the scheduling given by (7) is optimal. With this scheduling, QMF information flows through all cuts are equal to an optimal achievable QMF rate, $R_{Q M F}^{*}$. If we keep the same listening fractions $f_{i}^{*}$ but introduce overlaps, at least one cut will have its information flow reduced compared to $R_{Q M F}^{*}$, and the rate of the network will be reduced. If some listening fractions $f_{i}$ differ from those given by (5), we can identify at least one cut $C_{Y}$, such that $I_{C_{Y}}<R_{Q M F}^{*}$. With the new scheduling vector $\Lambda$ having $f_{i}>f_{i}^{*}$ where $i \in \mathcal{A}$ and $f_{j}<f_{j}^{*}$ where $j \in \mathcal{B}$, and $\mathcal{A} \cup \mathcal{B} \neq \emptyset$, any cut $C_{Y}$ that satisfies $\mathcal{A} \subset \mathcal{L}_{C_{X}}$ and $\mathcal{B} \subset \mathcal{R}_{C_{X}}$ will have information flow $I_{C_{Y}}<R_{Q M F}^{*}$. Any overlaps would further reduce the information flow through this cut.

We conclude that if the schedule given by (7) is feasible, it is optimal. Note that the number of non-zero scheduling parameters does not exceed $N+1$, as predicted by [3].

\section{Local Scheduling}

Theorem 1 leads to a very simple scheduling scheme that is based on (5). The idea is that each relay calculates the total listening fraction $f_{i}$ as if there were no other relays in the network by using only the channel state information (CSI) of the surrounding links: $S-D, S-R_{i}$ and $R_{i}-D$ (thus the name local scheduling). We have shown that under condition (6), these locally calculated listening fractions represent the optimal solution if the relays are scheduled such that there

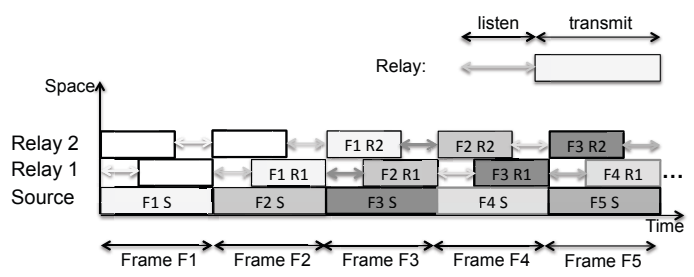

Fig. 2. DBLAST space-time coding

are no overlaps. If condition (6) is not satisfied, there may be some overlaps in listening between relays, and in general this scheme is no longer optimal.

We propose the following algorithm for local scheduling:

1) Each $f_{i}$ is calculated using (5);

2) If (6) is true then go to step 3), otherwise go to step 4);

3) Relays are scheduled such that there are no overlaps in listening;

4) Relay $R_{i}$ is scheduled to listen in interval $\left[\frac{2 i-1}{2 N}-\right.$ $\left.\frac{f_{i}}{2}, \frac{2 i-1}{2 N}+\frac{f_{i}}{2}\right]$, based on its index number $i$.

Through simulations, we verified that with insignificant loss in performance, we can choose fixed locations within the frame for listening intervals for each relay. With this algorithm, there is no need for computationally-intensive scheduling optimization, and we ensure that each relay switches between the listen and transmit phases only once per frame.

\section{Design Aspects of A QMF CoOperative RELAYING SYSTEM}

Approximation of the general network with the diamond network happens in two steps. First, by using a linear MIMO detector, we can represent multiple-access channel at the destination though a series of parallel channels. Second, we propose a scheme for canceling inter-relay interference that effectively eliminates relay-to-relay links from further analysis.

\section{A. DBLAST and MIMO Processing}

Diagonal Bell-Labs space-time (DBLAST) scheme [6] is shown in Figure 2 for the two-relay network. All relays listen according to their schedule during the source's transmission, and then delay their transmission by an integer number of frames. In particular, relay $R_{i}$ listens to the source's message $F_{k, S}$ during frame $F_{k}$, and transmits its message $F_{k, R_{i}}$ during frame $F_{k+i}$.

To detect all spatial streams at the destination, we apply the linear minimum mean-square error MIMO detector with successive interference cancellation (MMSE-SIC). The MMSESIC detection scheme turns the multiple-access channel at the destination into a series of parallel channels. The SNR values of these parallel channels are computed during the process of detection as: 


$$
\begin{aligned}
& I_{C_{X}}(\Lambda)=\sum_{\mathcal{S}_{C_{X}}^{L} \subset \mathcal{L}_{C_{X}}} \lambda_{\mathcal{S}_{C_{X}}^{L}} \log \left(1+S N R_{S D}\right)+\sum_{\substack{\mathcal{S}_{C_{X}}^{R} \subset \mathcal{R}_{C_{X}} \\
\left|\mathcal{S}_{C_{X}}^{R}\right| \geq 1}} \sum_{\mathcal{S}_{C_{X}}^{L} \subset \mathcal{L}_{C_{X}}} \lambda_{\mathcal{S}_{C_{X}}^{R} \cup \mathcal{S}_{C_{X}}^{L}} \log \left(1+S N R_{S D}+\sum_{i \in \mathcal{S}_{C_{X}}^{R}} \frac{S N R_{S R_{i}}}{2}\right) \\
& +\sum_{i \in \mathcal{L}_{C_{X}}}\left(1-f_{i}\right) \log \left(1+S I N R_{R_{i} D}\right)-\sum_{i \in \mathcal{L}_{C_{X}}} f_{i} \\
& I_{C_{X}}(\Lambda)=\log \left(1+S N R_{S D}\right)+\sum_{i \in \mathcal{R}_{C_{X}}} f_{i}\left[\log \left(1+S N R_{S D}+\frac{S N R_{S R_{i}}}{2}\right)-\log \left(1+S N R_{S D}\right)\right] \\
& +\sum_{i \in \mathcal{L}_{C_{X}}}\left(1-f_{i}\right) \log \left(1+S I N R_{R_{i} D}\right)-\sum_{i \in \mathcal{L}_{C_{X}}} f_{i}-\sum_{\substack{\mathcal{S}_{C_{X}}^{R} \subset \mathcal{R}_{C_{X}} \\
\left|\mathcal{S}_{C_{X}}^{R}\right| \geq 2}} \sum_{\mathcal{S}_{C_{X}}^{L} \subset \mathcal{L}_{C_{X}}} \lambda_{\mathcal{S}_{C_{X}}^{R} \cup \mathcal{S}_{C_{X}}^{L}} \times \Delta_{\mathcal{S}_{C_{X}}^{R}} \cup \mathcal{S}_{C_{X}}^{L}
\end{aligned}
$$

$$
\begin{aligned}
S N R_{S D} & =\left|\underline{h}_{S D}\right|^{2}=\underline{h}_{S D}^{H} \underline{h}_{S D} ; \\
S I N R_{R_{i} D} & =\underline{h}_{R_{i} D}^{H}\left(I+\underline{h}_{S D} \underline{h}_{S D}^{H}+\sum_{k=1}^{k=i} \underline{h}_{R_{k} D} \underline{h}_{R_{k} D}^{H}\right)^{-1} \underline{h}_{R_{i} D} .
\end{aligned}
$$

Destination processing of each frame consists of: 1) MIMO detection of the source's and the relays' streams related to information from frame $i$ (same-color messages related to frame $F_{i}$ in Figure 2) using the MMSE-SIC detector; 2) joint decoding of the detected streams as in [2], and 3) cancellation of the decoded streams from the received signals in frames $i+1, \ldots, i+N$.

\section{B. Inter-Relay Interference}

The QMF scheme described in [2] assumes that a relay receives only information from the source. In the general network scenario with multiple relays, a relay will receive a superposition of signals from the source and all relays that are in the transmit phase.

Estimating the source's message in the presence of (strong) interference from other relays would be detrimental for the performance of the system. In the rest of this section, we first explain the influence of the inter-relay interference, then we suggest a way to cancel it at the destination and establish the same type of relationship between source's and relays' messages as in [2]. This enables implementation of already developed decoding techniques from [2].

1) Effect of Inter-Relay Interference: In the general network configuration, the very first frame will experience no interrelay interference since the relays are still not transmitting (Figure 2). The received signal at relay $R_{i}$ can be expressed as:

$$
\begin{aligned}
& y_{R_{i}, 1}=h_{S R_{i}} x_{S, 1}+z \\
& y_{R_{i}, 1}^{\prime}=x_{S, 1}+z^{\prime},
\end{aligned}
$$

where $x_{T, i}$ denotes the transmitted symbol by terminal $T$ in frame $i, y_{T, i}$ denotes the received symbol at terminal $T$ in frame $i$, and $z$ is noise at the receiver. The quantized version of the received signal can be expressed as:

$$
Q\left(y_{R_{i}, 1}^{\prime}\right)=x_{S, 1}+z^{\prime}+z_{Q},
$$

where $z_{Q}$ is the quantization noise. For this frame, the relationship between the original source symbol, $x_{S, 1}$, and the quantized versions of this symbol at relay $R_{i}, Q\left(y_{R_{i}, 1}^{\prime}\right)$, is the same as it would be for a network without relay-torelay links. We denote this relationship between the source's message $F_{1, S}$ and the relays' messages $F_{1, R_{i}}$ in Figure 3 as a "Q" relationship. This relationship changes for subsequent frames because there is interference between relays. Figure 3 also presents relationships between messages from frame 3 and the previous frames, assuming the same two-relay system. The signals that relay $R_{i}$ receives during frame 3 and then forwards to the destination are respectively:

$$
\begin{aligned}
y_{R_{i}, 3} & =h_{S R_{i}} x_{S, 3}+h_{R_{j} R_{i}} x_{R_{j}, 3-j}+z ; \\
Q\left(y_{R_{i}, 3}^{\prime}\right) & =x_{S, 3}+x_{\text {interf }}^{\prime}+z^{\prime}+z_{Q} .
\end{aligned}
$$

The relationship between $x_{S, 3}$ and $Q\left(y_{R_{i}, 3}^{\prime}\right)$ is represented in Figure 3 as an "I" relationship. It suggests that additional information about frames 1 and 2 can be inferred, given some knowledge of the information from frame 3 . However, if the destination was to use this additional information about frames 1 and 2, all three frames would have to be decoded jointly. In the general network configuration with $N$ relays, $N+1$ frames would have to be decoded jointly. This would additionally increase the complexity of the decoding algorithm at the destination, and lead to a very high decoding latency.

2) Cancellation of Inter-Relay Interference: Instead of using additional information from the previous frames, we suggest canceling it as inter-relay interference. The interference from previous frames 1 and 2 can be cancelled before the joint decoding of the current frame 3 starts, since according to DBLAST these frames have already been decoded (Figure 2). In a general scenario with $N$ relays, the term $x_{\text {inter }}^{\prime}$ contains interference from $N$ previous frames that have all been decoded. Cancellation is always possible for any linear 

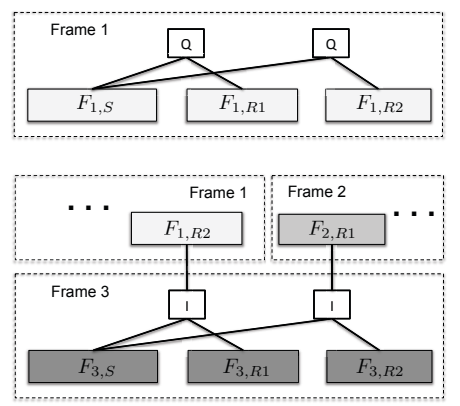

Fig. 3. Decoding of frames 1 and 3 for the two-relay general network.

encoding scheme at the relays, such as the one that was introduced in [2]. Effectively, (15) simplifies to (14), and relationship "I" simplifies to "Q".

After inter-relay interference cancellation is performed, the "I" relationship in any frame $i$ simplifies to the "Q" relationship, as if we had no interference in the first place. When applied to the general network from Figure 1, this algorithm can achieve the same rate as an equivalent network without relay-to-relay links under the same framework [2]. The loss of the suggested interference cancellation algorithm is exactly the difference between the QMF rates of these two networks. The benefit is that we maintained the same linear complexity and decoding latency as if there was no interference.

\section{Simulation Results}

In all simulations, the channels are assumed to have Rayleigh distribution with $\underline{h}_{T_{1} D} \sim \mathcal{C N}\left(0, S N R_{D} \times I\right)$ and $h_{T_{1} T_{2}} \sim \mathcal{C N}\left(0, S N R_{R}\right)$ where $S N R_{R}=S N R_{D}^{\eta}$, and the noise at each receive antenna is $z \sim \mathcal{C N}(0,1)$. The proximity gain $\eta$ comes from the assumption that relays are located much closer to the source and to each other than to the destination.

The simulations include computation of QMF rates both for the general network and the network without relay-to-relay links. In every channel instance, we applied both the optimal and the local scheduling scheme to both network types, then averaged computed QMF rates over many channel instances.

We show in Figure 4 how the achievable QMF rate scales with the number of relays $N$, for 6 receive antennas at the destination. Achievable QMF rate of a 5-relay system compared to a system without relays is approximately $2 \times$ higher for $\eta=2$ and $3 \times$ higher for $\eta=4$. We show that for the typical values of $\eta$, the achievable QMF rate of the network without relay-to-relay links is very close to that of the general network. Rate loss of the suggested inter-relay interference cancellation algorithm with 5 relays is around $2.5 \%$ and $5 \%$, for $\eta=2$ and $\eta=4$, respectively. Finally, we conclude that the local scheduling scheme performs close to the optimal one for small number of relays (up to 3 ).

In Fig 5, we show that the destination can successfully operate with less than 6 receive antennas. SNR values have been scaled such that the receive power gain is the same for all configurations, for fair comparison. We conclude that most of the multiplexing gain is captured even with 3 receive antennas,

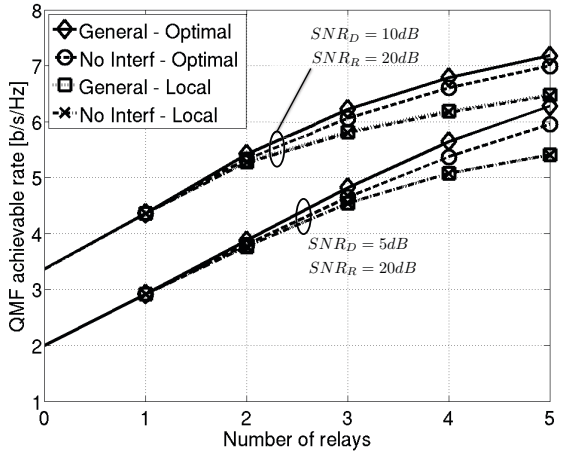

Fig. 4. QMF achievable rate scaling with the number of relays. Network with (General) and without (No Interf) relay-to-relay links, optimal and local relay scheduling.

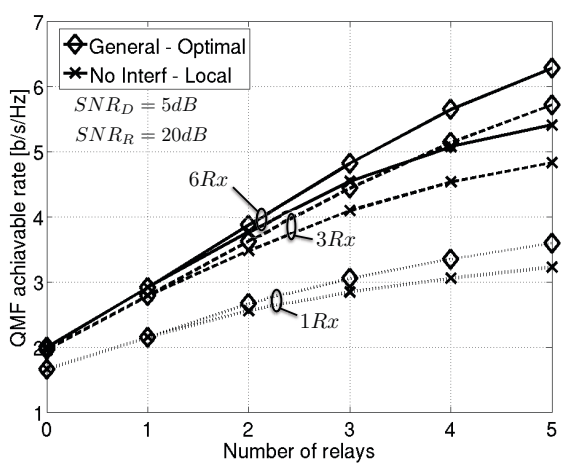

Fig. 5. QMF achievable rate scaling with the number of relays, for 6 (solid lines), 3 (dashed lines) and 1 (dotted lines) receive-antenna configurations

whereas 1 receive antenna captures only the power gain that comes from increasing number of transmit antennas.

\section{ACKNOWLEDGMENTS}

We acknowledge the students S. Ramakrishnan and K. Sun, faculty and sponsors of Berkeley Wireless Research Center, and support of the National Instruments Corporation, National Science Foundation (award EECS-1232318) and Center for Circuit \& System Solutions (C2S2) Focus Center, one of six research centers funded under the Focus Center Research Program, a Semiconductor Research Corporation program.

\section{REFERENCES}

[1] A. Avestimehr, S. Diggavi, and D. Tse, "Wireless network information flow: A deterministic approach," IEEE Trans. Info. Theory, 2011

[2] V. Nagpal, I. H. Wang, M. Jorgovanovic, D. Tse, and B. Nikolic, "Coding and system design for quantize-map-and-forward relaying," IEEE Journal on Selected Areas in Communications, 2013.

[3] S. Brahma, A. Ozgur, and C. Fragouli, "Simple schedules for half-duplex networks," in Proc. IEEE Int. Symp. Info. Theory, 2012.

[4] W. Wang, L. Ong, and M. Motani, "Transmission schedule optimization for half-duplex multiple-relay networks," in 7th Int. Symp. on Modeling and Optimization in Mobile, Ad Hoc, and Wireless Networks, 2009.

[5] S. H. Lim, Y.-H. Kim, A. El Gamal, and S.-Y. Chung, "Noisy network coding," IEEE Trans. Info. Theory, 2011.

[6] G. J. Foschini, "Layered space-time architecture for wireless communication in a fading environment when using multi-element antennas," Bell Labs Technical Journal, no. 2, pp. 41-59, 1996. 